\title{
Flavor at low and high $p_{T}$
}

\author{
Jernej. F. Kamenik* \\ Faculty of Mathematics and Physics, University of Ljubljana, Jadranska 19, 1000 Ljubljana, \\ Slovenia \\ Jožef Stefan Institute, Jamova 39, 1000 Ljubljana, Slovenia \\ E-mail: jernej.kamenikeijs.si
}

I briefly review the various aspects of the fruitful interplay between flavor and high- $p_{T}$ physics, especially in our ongoing quest to uncover physics beyond the standard model. After elucidating the power of indirect flavor bounds compared to the direct reach of particle colliders, the possibility of flavor safe new physics is discussed. A brief introduction on the flavor probes of the standard model Higgs sector is followed by a detailed account of the numerous intertwined ways in which the persistent experimental anomalies in charged and neutral current mediated B decays interface with direct searches for new degrees of freedom at the LHC. In particular, the counterintuitive notion that SM gauge invariance together with flavor constraints from kaon and charm sectors implies new physics well within the kinematical reach of the LHC and orders of magnitude below the naive effective theory considerations is explained. Explicit illustrative model examples are provided throughout.

18th International Conference on B-Physics at Frontier Machines - Beauty2019 -

29 September / 4 October, 2019

Ljubljana, Slovenia

${ }^{*}$ Speaker. 


\section{Introduction}

Historically, precision measurements and theoretical developments in flavor physics have many times successfully foreshadowed experimental discoveries in high energy particle colliders. Perhaps most well known are those of charm and top quark, whose existence and mass were successfully predicted based on observations of neutral kaon and $B$-meson oscillations, respectively. More generally, flavor and high- $p_{T}$ physics can interplay in several ways, especially in our ongoing quest for physics beyond the standard model (SM). For example low energy precision observables and direct searches at high $p_{T}$ often yield complementary constraints on new physics (NP) models. In addition, nontrivial flavor structure in the couplings of NP degrees of freedom to SM can affect collider signatures and consequently their reach. Finally, eventual experimental anomalies - deviations of measurements from SM expectations - in $B, D$ or kaon physics, can motivate NP searches at high $p_{T}$, and vice versa. In the following I elaborate on each of these aspects.

\section{Flavor bounds on new physics and the reach of the LHC}

From the theory perspective there exist several indications for the existence of new heavy degrees of freedom. Considering the running of the SM gauge couplings under the renormalization group evolution (RGE), the appealing idea of high scale gauge unification suggests new dynamics at or below the scales of the order $10^{(15-19)} \mathrm{GeV}$. In the foreseeable future, the only particle physics experiments with indirect sensitivity to such high scales will be those probing the proton lifetime. Much more promising are the scales indicated by models of thermal relic dark matter (DM), which typically predict new particles with masses below $10^{4} \mathrm{GeV}$. Interestingly, new degrees of freedom at similar scales are predicted by most mechanisms of electroweak (EW) scale stabilization against quantum corrections, addressing the so called EW hierarchy problem. This is the energy regime which is being actively explored by the LHC, where direct bounds on new heavy particle masses are often already reaching several $\mathrm{TeV}$. Flavor physics is in a somewhat paradoxical position in this respect. Theoretically, the flavor sector of the SM (with the exception of neutrino masses) is self contained and UV complete in the sense that it gives no indication of a scale at which new flavored degrees of freedom should arise. However, the observed patterns and hierarchies of the SM flavor parameters are highly suggestive of the existence of such new underlying dynamics. At the same time, precision measurements of flavor observables, especially flavor changing neutral current (FCNC) mediated processes generally exhibit good agreement with SM predictions and thus seem to exclude new degrees of freedom strongly coupling to flavor up to extremely high scales. In particular, we can parametrize the effects of such heavy dynamics on low energy observables in terms of an effective field theory power expansion:

$$
\mathscr{L}_{\mathrm{BSM}}=\mathscr{L}_{\mathrm{SM}}+\sum_{i,(d>4)} \frac{\mathscr{Q}_{i}^{(d)}}{\Lambda^{d-4}},
$$

where $\mathscr{L}_{S M}$ is the SM Lagrangian, $\mathscr{Q}_{i}^{(d)}$ are local operators of canonical dimension $d$, constructed out of SM fields, and respecting SM gauge symmetry, and $\Lambda$ is the effective cut-off scale of the theory. In the case of $\Delta F=2$ processes like neutral meson oscillations, the relevant operators first 
appear at dimension $d=6$ and are of the form

$$
\mathscr{Q}_{A B}^{(6)} \sim z^{i j}\left[\bar{q}_{i} \Gamma^{A} q_{j}\right] \otimes\left[\bar{q}_{i} \Gamma^{B} q_{j}\right],
$$

where the coefficients $z^{i j}$ parametrize the flavor structure of the couplings to the quark fields $q_{i, j}$ and $\Gamma^{A, B}$ denote elements of the Clifford algebra. Over the past few decades, there has been tremendous progress in this field on both the experimental and theoretical front. On the one hand, the Bfactories and more recently $\mathrm{LHCb}$ have performed neutral $D, B$ and $B_{s}$ meson oscillation and $\mathrm{CP}$ violation measurements with ever increasing precision. On the theory side, especially numerical methods of QCD on the lattice have allowed for ever more precise predictions of the relevant observables both within the SM and in presence of new contributions of the form (2.2). Today, the absence of any significant deviations from SM expectations can be interpreted in terms of bounds on the effective theory cut-off scale $\Lambda$. In particular, for generic CPV NP flavor structures $z^{i j} \sim$ $\left|z_{\mathrm{NP}}\right| \exp \left(i \phi_{\mathrm{NP}}\right)$ with $z_{\mathrm{NP}}, \phi_{\mathrm{NP}} \sim 1$, measurements of (CPV in) neutral kaon oscillations constrain $\Lambda \gtrsim 10^{6}\left(10^{8}\right) \mathrm{GeV}$. Measurements in the $D, B$ and $B_{s}$ sector currently yield somewhat weaker constraints, yet still exhibit indirect NP sensitivity far beyond the kinematical reach of the LHC (see e.g. Refs. [1, 2, 3]).

In fact, there are explicit NP model examples where new degrees of freedom are beyond direct kinematical reach, but can be probed with low energy precision measurements. One such case is the so called mini-split supersymmetry [4] - a version of the minimal supersymmetric standard model motivated by the measured value of the Higgs mass $\left(m_{h}\right)$. In particular, at the one loop order, one can write

$$
m_{h}^{2} \sim m_{Z}^{2} \cos ^{2} 2 \beta+\frac{3 m_{t}^{2}}{4 \pi^{2} v^{2}} \log \frac{m_{\tilde{t}}^{2}}{m_{t}^{2}}
$$

where $m_{Z, t, \tilde{t}}$ denote masses of the SM $Z$ boson, the top quark, and its scalar (supersymmetric) partner, the $\tilde{t}$ squark, while $v=246 \mathrm{GeV}$ and $\tan \beta$ denotes the ratio of the two Higgs condensates in the model. One can observe that depending on the value of $\beta$, accommodating the measured Higgs mass requires $m_{\tilde{t}}$ in the range from $10 \mathrm{TeV}$ to $10^{5} \mathrm{TeV}$ and beyond. Interestingly even such high new particle thresholds (up to $\mathrm{PeV}$ ) can be probed with low energy flavor observables provided the squark mass matrices are of generic form $m_{Q}^{2}=m_{\tilde{q}}^{2}\left(\mathbf{1}+\delta_{q}^{L}\right)$ with $\left(\delta_{q}^{L}\right)_{i j} \sim \mathscr{O}(1)$. What is more, significant improvements are expected in several of the measurements, most sensitive to this kind of new flavor dynamics, in the next decade.

\section{Flavor safe new physics?}

Of course, the assumption of generic flavor violation in the NP sector might be too optimistic. But can one conversely construct NP models, completely transparent to low energy flavor probes? The main theoretical objection to such a construction lies in the fact that flavor is already broken in the SM by the presence of the Higgs Yukawa couplings. This implies for example that any additional scalar coupling to SM fermions necessarily introduces additional breaking, which can at most be aligned with that of the SM Higgs. On the other hand, new massive vectors coupling to SM fermionic currents can preserve flavor. In both cases however, NP contributions to FCNCs do not vanish, but are loop and GIM suppressed, as in the SM (see e.g. Ref. [5]). This limit often goes under the label of minimal flavor violation (MFV) [6]. 
A typical example of this kind are simplified DM models with (pseudo)scalar mediators. Focusing for concreteness on a fermionic DM field $(\chi)$ coupled to the SM via a pseudoscalar field $(A)$, the relevant interaction terms are

$$
\mathscr{L}_{\mathrm{DM}} \ni i g_{\chi} A \bar{\chi} \gamma^{5} \chi+\sum_{f=q, \ell, v} i g_{f} A \bar{f} \gamma^{5} f
$$

where $g_{\chi, f}$ denote the relevant couplings. An important feature of this model is that the pseudoscalar nature of the DM-SM interactions leads to suppressed signals in direct DM detection experiments. Furthermore, if the $\chi, A$ masses satisfy the relation $m_{\chi}>m_{A} / 2$, direct DM production at particle colliders is suppressed even if $\chi$ is well within the kinematical range. In this case, some of the most stringent bounds on the model can be derived from flavor observables. The MFV limit of SM Yukawa-like couplings is reached for $g_{f}=\sqrt{2} g_{Y} m_{f} / v$, where $g_{Y}$ is now a flavor universal coupling parameter. Even in this limit FCNC processes with dimuon final states dominate the bounds at low $m_{A}$ where $A$ can appear as narrow peak in the di-muon distribution [7]. Once $A$ becomes too heavy to be produced in hadron decays, low mass di-muon searches at LHC(b) provide the most sensitive probe up to $m_{A} \gtrsim 50 \mathrm{GeV}[8,9]$, when other high- $p_{T}$ searches in final states like $\gamma \gamma, t \bar{t}$ and $t \bar{t} t \bar{t}$ become the dominant probes [10,11].

\section{Flavor probes of the Higgs sector}

The generation of fermion masses in the SM through the Higgs mechanism leads to distinct predictions for the flavor structure of Higgs Yukawa couplings to fermions $\left(\mathscr{L} \ni y_{f} h \bar{f} f\right)$. In particular the exact relation

$$
y_{f}^{\mathrm{SM}}=\frac{\sqrt{2} m_{f}}{v},
$$

has several important features, which can be experimentally tested [12], in particular (a) proportionality $y_{i i} \propto m_{i}$ with a precisely determined proportionality factor (b) $y_{i i} / m_{i}=\sqrt{2} / v$, see e.g. Ref. [13]; (c) diagonality $y_{i j}=0$ for $i \neq j$, see e.g. Refs. [14, 15]; and finally (d) the CP nature, namely the absence of the $h \bar{f} \gamma_{5} f$ terms, see e.g. Refs. [16, 17, 18]. There has been tremendous activity on both the theoretical and experimental side to probe these SM predictions at ever increasing precision.

\section{Flavor anomalies motivate high- $p_{T}$ searches}

Over the past eight years, experiments probing lepton flavor universality (LFU) in charged current mediated semileptonic $b$-hadron decays have persistently reported results at variance with SM expectations. In particular, ratios of decay rates

$$
R\left(D^{(*)}\right)=\frac{\Gamma\left(B \rightarrow D^{(*)} \tau \nu\right)}{\Gamma\left(B \rightarrow D^{(*)} \ell v\right)}
$$

with $\ell=e, \mu$, are well understood theoretically, since most of the hadronic (form factor) uncertainties cancel or can be reduced using lattice QCD inputs and/or heavy quark effective theory 
calculations [19]. On the other hand, the measured values of these ratios exhibit a combined tension of about $\mathscr{O}(3 \sigma)$ with respect to the theoretical predictions within the SM, indicating an almost universal $\mathscr{O}(20 \%)$ upward deviation [20, 21].

Intriguingly, departures from LFU are also observed in FCNC mediated rare semileptonic B decays. The corresponding LFU ratios

$$
R_{K^{(*)}}=\frac{\int d q^{2}\left[d \Gamma\left(B \rightarrow K^{(*)} \mu^{+} \mu^{-}\right) / d q^{2}\right]}{\int d q^{2}\left[d \Gamma\left(B \rightarrow K^{(*)} e^{+} e^{-}\right) / d q^{2}\right]}
$$

are defined by integrating over a particular bin of the lepton pair invariant mass squared $\left(q^{2}\right)$. Crucially, the integration ranges should be identical for both the numerator and denominator. In this case the expected departures from $\operatorname{LFU}\left(R_{K^{(*)}}=1\right)$ within the SM are expected to be at the subpercent level away from kinematical thresholds. In contrast, recent experimental results by LHCb seem to indicate an $\mathscr{O}(20 \%)$ downward deviation with a significance of about $\mathscr{O}(2.5 \sigma)[22,23]$. If interpreted in terms of NP contributions to the relevant $\left[\bar{s} \Gamma^{A} b\right] \otimes\left[\bar{\mu} \Gamma^{B} \mu\right]$ effective operators, these indications are strengthened by corroborating evidence coming from $B \rightarrow K^{*} \mu^{+} \mu^{-}$angular analyses and rate measurements of various $b \rightarrow s \mu^{+} \mu^{-}$mediated decays [24].

Immediately, some general implications of these so called $B$-anomalies for direct searches at high $p_{T}$ can be derived in the following way: as discussed in Sec. 2, heavy NP effects in low energy observables can be parametrized in full generality using the power series in Eq. (2.1). Consequently, current NP indications in $B$-decays can be phrased in terms of preferred values for the NP scale $\Lambda$. This however does not guarantee the existence of new degrees of freedom at that scale. On dimensional grounds namely, an energy scale is related to a particle mass threshold via the relation

$$
[\text { scale }]=\frac{[\text { mass }]}{[\text { coupling }]} .
$$

Thus, while without knowledge of the UV theory with its predictions for the relevant couplings, no lower bound can be set on the new particle masses, an upper bound can be derived using arguments of perturbative unitarity or pertubativity [25]. In particular, deviations in $R\left(D^{(*)}\right)$ typically imply $\Lambda \simeq 2.5 \mathrm{TeV}$ (e.g. for the operator $\mathscr{Q}=\left(\bar{c}_{L} \gamma_{\mu} \bar{b}_{L}\right)\left(\bar{\tau}_{L} \gamma^{\mu} v_{L}\right)$ ), which when combined with unitarity arguments yields $m_{\mathrm{NP}} \lesssim 6.5 \mathrm{TeV}$ for the relevant NP particle mass threshold, uncomfortably close to the edge of the LHC kinematical reach (see e.g. Refs. [26, 27]). Conversely deviations in $R_{K^{(*)}}$ typically imply even a much larger scale $\Lambda \simeq 40 \mathrm{TeV}$ (e.g. for $\mathscr{Q}=\left(\bar{s}_{L} \gamma_{\mu} \bar{b}_{L}\right)\left(\bar{\mu}_{L} \gamma^{\mu} \mu_{L}\right)$ ) meaning that the corresponding NP degrees of freedom could only be accessible at the LHC, if their couplings to $\bar{s} b$ and/or $\bar{\mu} \mu$ are suppressed.

More specific implications can be derived by employing the known symmetry structure of the SM. In particular, any heavy NP needs to respect the SM gauge symmetry. The relevant effective operators thus need to be constructed from gauge covariant fermionic fields (in particular $Q, D, U$, $L$ and $E$, denoting the left handed $S U(2)_{L}$ quark doublets, right-handed down and up quark weak singlets, left handed lepton doublets and right-handed charged lepton weak singlets, respectively). This in turn leaves only a few possibilities which can account for apparent LFU violations and at the same time be consistent with other kinematical properties measured in these decays, these 
include $^{1}$

$$
\begin{array}{lr}
\varepsilon_{i j}^{L} \varepsilon_{k l}^{Q}\left(\bar{L}_{i} L_{j}\right)\left(\bar{Q}_{k} Q_{l}\right), & \varepsilon_{i j}^{E L} \varepsilon_{k l}^{Q D}\left(\bar{E}_{i} H^{\dagger} L_{j}\right)\left(\bar{Q}_{k} H D_{l}\right), \\
\varepsilon_{i j}^{E} \varepsilon_{k l}^{Q}\left(\bar{E}_{i} E_{j}\right)\left(\bar{Q}_{k} Q_{l}\right), & \varepsilon_{i j}^{L E} \varepsilon_{k l}^{Q U}\left(\bar{L}_{i} H E_{j}\right)\left(\bar{Q}_{k} \tilde{H} U_{l}\right),
\end{array}
$$

where $H$ is the SM Higgs weak doublet and $\tilde{H}_{i}=\varepsilon_{i j} H_{j}$. Not all of these operators can however accommodate both charged and neutral current $B$-anomalies. In particular, those on the right hand side, which are typically generated in multi-Higgs doublet SM extensions, can only address $R\left(D^{(*)}\right)$. They are however also severely constrained by the measurements of the $B_{c}$ lifetime (see e.g. Refs. [31, 32, 33]). Conversely, the operators on the left-hand side can address the $R_{K^{(*)}}$ anomaly. Interestingly, non-vanishing $\varepsilon_{\mu \mu}^{L}, \varepsilon_{\tau i}^{L}, \varepsilon_{s b}^{Q}$ and $\varepsilon_{c b}^{Q}$ can in principle simultaneously address both $B$-anomalies in terms of a single operator (on the top left). These operators are typically generated through new massive vector boson $\left(W^{\prime} / Z^{\prime}\right)$ and/or leptoquark exchanges in gauge symmetry extensions of the SM.

In the case of the operators (and their corresponding simplified UV completions) addressing the $R\left(D^{(*)}\right)$ anomaly, a simple application of crossing symmetry allows to derive severe constraints from measurements of high $p_{T}$ mono-tau production at the LHC [30]. In particular while the partonic cross-section $\hat{\sigma}(b \bar{c} \rightarrow \tau \bar{v})$ is severely diluted in proton-proton collisions by the smallness of the relevant $c$ - and $b$-quark parton distribution functions, this is compensated by its growth with the partonic invariant mass $\hat{\sigma} \propto\left(\hat{s} / m_{\mathrm{NP}}^{2}\right)^{2}$ below the mediator mass. The combined effect is pronounced enough to already exclude or severely constrain several of the relevant NP scenarios.

Further insight can be obtained by accounting for the absence of experimental indications for anomalous LFU violations and FCNCs in other flavor transitions, as probed by Kaon, Charm, Tau decay measurements. This implies an approximate alignment of the new flavor structures $\left(\varepsilon_{i j}^{Q, Q D, Q U}\right)$ with the 3 rd quark generation, leading to an approximate $U(2)$ flavor symmetry or in the extreme limit, to MFV in which $\varepsilon_{s b}^{Q} \propto V_{t b} V_{t s}$ and $\varepsilon_{c b}^{Q} \propto V_{c b}$ [28, 34]. One would be tempted to interpret these results as an indication of approximately flavor universal NP flavor structure of the form

$$
\varepsilon^{Q} \simeq \mathbf{1}+\mathscr{O}\left(\mathbf{Y} \mathbf{Y}^{\dagger}\right)
$$

where $\mathbf{Y}$ denotes the SM quark Yukawa matrices. However this possibility predicts (flavor) unsuppressed contributions to $\bar{u} u, \bar{d} d \rightarrow \mu^{+} \mu^{-}$and is already excluded by LHC measurements of the high $p_{T}$ dimuon spectrum. Another important implication of flavor alignment is that it effectively lowers the relevant NP mass scale as indicated by the $B$-anomalies. In particular starting from the fermionic field structure in the 3rd generation alignment limit $\left(\bar{Q}_{3} Q_{3}\right)\left(\bar{L}_{3} L_{3}\right)$ the relevant operator contributing to $R\left(D^{(*)}\right)$ is now of the form $V_{c b}\left(\bar{c} \gamma_{\mu} b\right)\left(\bar{\tau} \gamma^{\mu} v\right)$ and the anomaly translates to an effective scale $\Lambda \sqrt{\left|V_{c b}\right|} \sim 0.5 \mathrm{TeV}$, well within the LHC reach. A similar exercise applied to the $R_{K^{(*)}}$ anomaly, starting from the structure $\left(\bar{Q}_{3} Q_{3}\right)\left(\bar{L}_{2} L_{2}\right)$ yields $\Lambda \sqrt{\left|V_{t s}\right|} \sim 8 \mathrm{TeV}$, and is thus still only marginally accessible at the LHC. Thirdly, flavor alignment applied to $R\left(D^{(*)}\right)$ predicts LFU violation in top decays, as can be easily seen by expanding $\left(\bar{Q}_{3} Q_{3}\right)\left(\bar{L}_{3} L_{3}\right) \rightarrow V_{c b}(\bar{c} b)(\bar{\tau} v)+V_{t b}(\bar{t} b)(\bar{\tau} v)$. Experimentally, LFU has been tested in the dominant top quark decay channel $t \rightarrow b(W \rightarrow \ell v)$ to

\footnotetext{
${ }^{1}$ Additional possibilities consist of so called tensor operators and operators constructed by including light new fermionic fields, which are complete singlets under the SM gauge group [28, 29]. Both are only relevant for the $R\left(D^{(*)}\right)$ anomaly, the later already being excluded by mono-tau searches at the LHC [30], see below.
} 
approximately $10 \%$ precision [35], which is sufficient to already constrain NP models addressing $R\left(D^{(*)}\right)$ through light $\left(m_{N P}<m_{t}-m_{b}\right)$ mediators [36]. More interestingly, in the future, measurements exploiting the different kinematics of the SM and NP contributions to $t \rightarrow b \tau v$, by measuring the differential LFU ratio as a function of the $b$-jet energy, could potentially probe LFU in top decays down to sub-percent level.

While non-universal flavor alignment models can avoid the most severe constraints derived from high- $p_{T}$ di-lepton searches, in the case of $R\left(D^{(*)}\right)$, this not enough, since SM gauge invariance implies the existence of unsuppressed $\left(\bar{Q}_{3} Q_{3}\right)\left(\bar{L}_{3} L_{3}\right) \rightarrow \ldots+(\bar{b} b)(\bar{\tau} \tau)$ operator structures leading to neutral current mediated $p p \rightarrow \tau^{+} \tau^{-}$production at the LHC [37]. In this strict alignment limit (see also Ref. [38]), constraints from existing di-tau resonance searches and spectrum measurements basically exclude most $R\left(D^{(*)}\right)$ explanations with charged scalars (including scalar leptoquarks). On the other hand, $W^{\prime}$ scenarios can still avoid these constraints but only if the corresponding $Z^{\prime}$ resonance is either light $\left(m_{Z^{\prime}} \lesssim 400 \mathrm{GeV}\right)$ and/or extremely broad $\left(\Gamma_{Z^{\prime}} / m_{Z^{\prime}} \gtrsim 20 \%\right)$ implying questionable perturbative control of the theory. Again, future LHC measurements with larger statistics are expected to further constrain the parameter space of the remaining possibilities.

Somewhat surprisingly, it turns out that the theoretical conundrum of non-universal flavor alignment can in the case of $R_{K^{(*)}}$ be solved quite elegantly through EW radiative effects, again with important implications for high- $p_{T}$ searches. Starting from manifestly flavor conserving but non-universal operator structures of the form

$$
\left(\bar{L}_{2} L_{2}\right)\left(\bar{U}_{3} U_{3}\right), \quad\left(\bar{E}_{2} E_{2}\right)\left(\bar{U}_{3} U_{3}\right)
$$

at the NP scale, EW matching and RGE, driven by top quark and $W$ loops, induce LFU violation in rare FCNC $B$ decays of exactly 3rd generation aligned (MFV) type, without the dangerous flavor universal term in Eq. (5.6) [39, 40]. Correspondingly, the effective NP scale is now loop-suppressed implying new degrees of freedom below $\Lambda \sqrt{\left|V_{t s}\right|} / 4 \pi \sim 600 \mathrm{GeV}$ and thus well within the LHC reach. However, which are these low lying new particles? An illustrative example is provided by a simple UV completion of the effective theory in terms of the vector-like top partner, charged under a new $U(1)^{\prime}$ gauge symmetry [41]. After its Higgsing, the SM top quark acquires a small effective $U(1)^{\prime}$ charge through mass mixing and thus couples to the now massive $U(1)^{\prime}$ vector boson, the $Z^{\prime}$. Coupling of $Z^{\prime}$ to muons can be generated in a similar way, eventually leading to effective operators with the structure in Eq. (5.7) below the $Z^{\prime}$ mass scale. The dominant signatures at high $p_{T}$ turn out to be quite distinctive in the form of non-resonant four top quark production [42] and associated $p p \rightarrow \mu^{+} \mu^{+} t \bar{t}$ production (see also Ref. [43]). Since the lowest lying new degrees of freedom (the $Z^{\prime}$ ) are now well within the LHC reach and have significant couplings to SM (to tops and muons) existing searches are already probing parts of the relevant parameter space and should completely cover it in the future.

\section{Conclusions}

Flavor remains a powerful guide to high- $p_{T}$ searches in the era of the LHC. In case new phenomena are discovered at high energies, flavor physics will allow to disentangle different possible interpretations and discriminate between different proposals and scenarios. The SM Higgs boson is 
a prime example of this. Conversely, in case no new degrees of freedom are seen at the LHC, precision tests of flavor, $\mathrm{CP}$, as well as baryon and lepton numbers will possibly remain our best probes forward. Their sensitivity in many cases already (far) exceeds energies/scales attainable in present and planned particle colliders and even cosmic ray observations. Similarly, in case of significant signals of NP in flavor observables, it is often possible to identify prospective LHC experimental targets. Unfortunately, NP degrees of freedom accommodating present tentative $B$-anomalies could well be beyond the LHC reach. Phenomenological and model-building considerations however point towards more optimistic scenarios. Somewhat counter-intuitively, low energy constraints in this case can lead us to consider lighter mediators, with distinct signatures at high $p_{T}$. A truly striking demonstration of the very fruitful interplay between NP searches at the high energy and intensity frontiers.

\section{References}

[1] A. Bevan et al., arXiv:1411.7233 [hep-ph].

[2] N. Carrasco et al. [ETM Collaboration], Phys. Rev. D 92, no. 3, 034516 (2015)

[3] A. Bazavov et al., Phys. Rev. D 97, no. 3, 034513 (2018)

[4] W. Altmannshofer, R. Harnik and J. Zupan, JHEP 1311, 202 (2013)

[5] J. F. Kamenik and C. Smith, JHEP 1203, 090 (2012)

[6] G. D’Ambrosio, G. F. Giudice, G. Isidori and A. Strumia, Nucl. Phys. B 645, 155 (2002)

[7] M. J. Dolan, F. Kahlhoefer, C. McCabe and K. Schmidt-Hoberg, JHEP 1503, 171 (2015) Erratum: [JHEP 1507, 103 (2015)]

[8] U. Haisch and J. F. Kamenik, Phys. Rev. D 93, no. 5, 055047 (2016)

[9] R. Aaij et al. [LHCb Collaboration], JHEP 1809, 147 (2018)

[10] C. Arina et al., JHEP 1611, 111 (2016)

[11] S. Banerjee, D. Barducci, G. Bélanger, B. Fuks, A. Goudelis and B. Zaldivar, JHEP 1707, 080 (2017)

[12] A. Dery, A. Efrati, Y. Hochberg and Y. Nir, JHEP 1305, 039 (2013)

[13] G. Perez, Y. Soreq, E. Stamou and K. Tobioka, Phys. Rev. D 92, no. 3, 033016 (2015)

[14] G. Blankenburg, J. Ellis and G. Isidori, Phys. Lett. B 712, 386 (2012)

[15] R. Harnik, J. Kopp and J. Zupan, JHEP 1303, 026 (2013)

[16] R. Harnik, A. Martin, T. Okui, R. Primulando and F. Yu, Phys. Rev. D 88, no. 7, 076009 (2013)

[17] J. Brod, U. Haisch and J. Zupan, JHEP 1311, 180 (2013)

[18] J. Ellis, D. S. Hwang, K. Sakurai and M. Takeuchi, JHEP 1404, 004 (2014)

[19] T. Kitahara, these proceedings.

[20] M. Tilley, these proceedings.

[21] K. Hara, these proceedings.

[22] C. Langenbruch, these proceedings. 
[23] S. Watanuki, these proceedings.

[24] S. Descotes-Genon, these proceedings.

[25] L. Di Luzio and M. Nardecchia, Eur. Phys. J. C 77, no. 8, 536 (2017)

[26] W. Altmannshofer, P. S. Bhupal Dev and A. Soni, Phys. Rev. D 96, no. 9, 095010 (2017)

[27] S. Iguro, Y. Omura and M. Takeuchi, Phys. Rev. D 99, no. 7, 075013 (2019)

[28] S. Fajfer, J. F. Kamenik, I. Nisandzic and J. Zupan, Phys. Rev. Lett. 109, 161801 (2012)

[29] A. Greljo, D. J. Robinson, B. Shakya and J. Zupan, JHEP 1809, 169 (2018)

[30] A. Greljo, J. Martin Camalich and J. D. Ruiz-Álvarez, Phys. Rev. Lett. 122, no. 13, 131803 (2019)

[31] R. Alonso, B. Grinstein and J. Martin Camalich, Phys. Rev. Lett. 118, no. 8, 081802 (2017)

[32] A. G. Akeroyd and C. H. Chen, Phys. Rev. D 96, no. 7, 075011 (2017)

[33] M. Blanke, A. Crivellin, S. de Boer, T. Kitahara, M. Moscati, U. Nierste and I. Niĺandÿi?, Phys. Rev. D 99, no. 7, 075006 (2019)

[34] M. Bordone, G. Isidori and S. Trifinopoulos, Phys. Rev. D 96, no. 1, 015038 (2017)

[35] G. Aad et al. [ATLAS Collaboration], Phys. Rev. D 92, no. 7, 072005 (2015)

[36] J. F. Kamenik, A. Katz and D. Stolarski, JHEP 1901 (2019) 032

[37] D. A. Faroughy, A. Greljo and J. F. Kamenik, Phys. Lett. B 764, 126 (2017)

[38] D. Buttazzo, A. Greljo, G. Isidori and D. Marzocca, JHEP 1711, 044 (2017)

[39] J. Aebischer, A. Crivellin, M. Fael and C. Greub, JHEP 1605, 037 (2016)

[40] J. E. Camargo-Molina, A. Celis and D. A. Faroughy, Phys. Lett. B 784, 284 (2018)

[41] J. F. Kamenik, Y. Soreq and J. Zupan, Phys. Rev. D 97, no. 3, 035002 (2018)

[42] E. Alvarez, D. A. Faroughy, J. F. Kamenik, R. Morales and A. Szynkman, Nucl. Phys. B 915,19 (2017)

[43] P. J. Fox, I. Low and Y. Zhang, JHEP 1803, 074 (2018) 